\title{
Vaccinia virus GLV-1h237 carrying a Walker A motif mutation of mouse Cdc6 protein enhances human breast tumor therapy in mouse xenografts
}

\author{
ELISABETH HOFMANN ${ }^{1}$, FRIEDRICH GRUMMT ${ }^{1}$ and ALADAR A. SZALAY FI,2,3,4,5 $^{1}$
}

\author{
${ }^{1}$ Department of Biochemistry, Biocenter, University of Würzburg, Würzburg, Germany; ${ }^{2}$ Genelux Corporation, \\ San Diego Science Center, San Diego, CA, USA; ${ }^{3}$ Virchow Center for Experimental Biomedicine, and ${ }^{4}$ Institute for \\ Molecular Infection Biology, University of Würzburg, Würzburg, Germany; ${ }^{5}$ Department of Radiation Oncology, \\ Moores Cancer Center, University of California, San Diego, La Jolla, CA, USA
}

Received September 22, 2010; Accepted November 23, 2010

DOI: $10.3892 /$ ijo. 2011.910

\begin{abstract}
Recently it was shown that recombinant vaccinia virus GLV-1h68 is a promising tool for treating different type of cancers in animal models. The goal of the present study was to enhance the oncolytic potential of GLV-1h68 without decreasing its safety. A derivative of GLV-1h68 containing the gene for a Walker A motif mutant of the essential cell cycle protein Cdc6, GLV-1h237, was engineered. The characteristics of GLV-1h237 and its efficiency in treating human breast cancer GI-101A cells were compared with that of GLV-1h236 (carrying the wild-type gene for Cdc6), GLV-1h71 (a derivative of GLV-1h68) and GLV-1h68, respectively. RT-PCR and immunoblot analyses revealed that Cdc6 is efficiently overexpressed in GLV-1h237-infected GI-101A cells. GLV-1h237 was found to have higher replication efficiency and enhanced cytotoxity than GLV-1h68 in cell culture. In the GI-101A tumor xenograft animal model, GLV-1h237 turned out to be the most potent oncolytic virus strain investigated. A single i.v. injection of GLV-1h237 resulted in enhanced anti-tumor activity compared to GLV1 h68 concomitant with a high tumor selectivity and a comparable safety profile. Thus, the strategy to combine oncolytic virotherapy with agents that interfere with host cell DNA synthesis is a promising approach for effective cancer therapy.
\end{abstract}

\section{Introduction}

Oncolytic viruses have a promising potential in treating tumors and are therefore currently investigated intensively. These

Correspondence to: Dr Aladar A. Szalay, Genelux Corporation, San Diego Science Center, 3030 Bunker Hill Street, San Diego, CA 92109, USA

E-mail: aaszalay@genelux.com

Key words: oncolytic virotherapy, vaccinia virus, cell division cycle 6, DNA replication, cell cycle, breast cancer viruses, either naturally occurring or genetically engineered, have the ability to infect, replicate in and specifically kill cancer cells either after intratumoral or systemic administration. Among oncolytic viruses used both in research and clinical studies are adenovirus $(1,2)$, measles virus $(3,4)$, vesicular stomatitis virus $(5,6)$, Newcastle disease virus $(7,8)$, reovirus $(9,10)$, herpes simplex virus $(11,12)$ and vaccinia virus $(13,14)$. Vaccinia virus (VACV) has great advantages as an oncolytic agent because of its broad host range, the efficient replication exclusively in the cytoplasm of host cells, the natural tropism for tumor tissues, and its large genome allowing the insertion of multiple foreign genes $(13,15)$. Furthermore, the remarkable safety record due to its historic use as a vaccine in the eradication of smallpox is another attractive hallmark of VACV. In several studies, tumor-specific replication and effective anti-tumor activities were observed when mice bearing human or murine tumor xenografts were treated with attenuated VACV strains, some of them additionally carrying diagnostic or therapeutic genes (16-20). Recently it was shown that a triple deletion of the $F 14.5 L, J 2 R$ (encoding thymidine kinase) and A56R (encoding hemagglutinin) loci in the LIVP strain of VACV reduced toxicity and enhanced tumor targeting specificity compared with its parental LIVP strains (21). Treatment with this virus, GLV-1h68, leads to inhibition of tumor growth in human breast cancer (21), anaplastic thyroid carcinoma (22), malignant pleural mesothelioma xenografts (23), squamous cell carcinoma (24), pancreatic carcinomas (25), prostate carcinomas (26) as well as canine mammary adenoma (27) and carcinoma (28).

Cell division cycle 6 (Cdc6) is essential for initiation of DNA replication in eukaryotic cells by playing an important role in the assembly of pre-replicative complexes (pre-RCs) at the origins of replication. During G1 phase of the cell cycle, pre-RC assembly starts by the binding of the originrecognition complex (ORC) to replication origins followed by the loading of Cdc6. Together with Cdt1, Cdc6 then recruits multiple copies of the minichromosome maintenance (MCM) protein complex, thereby forming the pre-RCs. Transition into $\mathrm{S}$ phase is initiated by activation of two $\mathrm{S}$ phase- 
Table I. Primer sequences.

\begin{tabular}{lllc}
\hline Gene & \multicolumn{1}{c}{ Forward (5'-3') } & \multicolumn{1}{c}{ Reverse (5'-3') } & Product size (bp) \\
\hline$m C d c 6$ & GTC AGG AGC CAG ACA GTC CT & TGC CGT TCA GGA CAT GCT CTA TTT C & 430 \\
$h C d c 6$ & ACGTCTGGGCGATGACAACC & CTG GCA GGC AGC CGA TC & 354 \\
lacZ & GGC GAG TTG CGT GAC TAC C & CAC GGC GTT AAA GTT GTT CTG CTT C & 419 \\
$\beta$-actin & GGA GAA AAT CTG GCA CCA CAC C & CCA TCT CTT GCT CGA AGT CCA GG & 437 \\
\hline
\end{tabular}

specific protein kinases and is marked by the conversion of pre-RCs to initiation complexes $(29,30)$.

Cdc6 is a member of the AAA $^{+}$family, i.e. ATPases associated with various cellular activities $(30,31)$. Like other members of this family, Cdc6 contains an NTP binding site consisting of conserved Walker A (GXXGXGKT) and Walker B (DEXD) motifs suggesting that ATP binding and hydrolysis regulate Cdc6 activity. The Walker A motif contains an invariant lysine residue and is essential for phosphate binding of ATP, whereas the acidic residues within the Walker B motif coordinate a magnesium ion and are crucial for ATP hydrolysis (32-34). Mutations in the Walker A motifs of yeast $(35,36)$, mammalian $(37,38)$ and Xenopus Cdc6 (39) led to failures in the assembly of pre-RCs and, as a consequence therefore, initiation of DNA replication is impaired in these cells.

It has been reported that levels of replication proteins like Cdc6, Cdt1 or MCM proteins are upregulated in different types of cancer compared to their normal counterparts (40-44) and therefore it has been suggested that they might serve as tumor diagnostic markers. Despite the use as a biomarker in cancer diagnostics, the overexpression of replication proteins in tumors also provides a promising opportunity in cancer therapy by creating agents that specifically target replication proteins.

The goal of the present study was to further enhance the promising anti-tumor activity of GLV-1h68 without decreasing its safety. Our idea was to generate a new recombinant VACV, starting from a derivative of GLV-1h68, overexpressing the essential cell cycle protein Cdc6 containing a mutated Walker A motif. The capability of the resulting virus, GLV-1h237, to infect, lyse and replicate in cell cultures of human breast cancer cells and to treat human GI-101A breast tumor xenografts in live animals was investigated. Our results suggest that GLV-1h237 has a stronger therapeutic potential against human breast cancer in nude mice concomitant with unchanged safety profile compared to the previously described GLV-1h68 (21).

\section{Materials and methods}

Cell lines. African green monkey kidney fibroblasts (CV-1; ATCC number CCL-70) were cultured in growth medium consisting of DMEM with $10 \%$ fetal bovine serum (FBS; PAA, Pasching, Austria) and antibiotic solution (100 U/ml penicillin $\mathrm{G} / 100 \mathrm{U} / \mathrm{ml}$ streptomycin; PAA) at $37^{\circ} \mathrm{C}$ under $5 \%$ $\mathrm{CO}_{2}$. The GI-101A cell line (21) was maintained in RMPI1640 (PAA) supplemented with 20\% FBS, 10 mM HEPES, antibiotic solution (all from PAA), $1 \mathrm{mM}$ sodium pyruvate, $5 \mathrm{ng} / \mathrm{ml} \beta$-estradiol and $5 \mathrm{ng} / \mathrm{ml}$ progesterone (all from Sigma-Aldrich, Steinheim, Germany) at $37^{\circ} \mathrm{C}$ and $5 \% \mathrm{CO}_{2}$.

Cloning and generation of vaccinia viruses expressing wildtype and mutant Cdc6. MmCdc6 cDNA was obtained from F. Grummt's laboratory. The conserved lysine residue 237 within the Walker A motif of MmCdc6 was replaced by glutamate using site-directed mutagenesis (cdc6-K237Eforw: 5'-GCTCCTGGGACTGGAGAAACTGCCTGTTTAA GCCG-3'; cdc6-K237E-rev: 5'-CGGCTTAAACAGGCAGT TTCTCCAGTCCCAGGAGC-3') (45). Wild-type and mutant cDNAs were cloned into transfer plasmids containing flanking regions of the VACV A56R locus. To generate recombinant VACV, CV-1 cells were infected with $1 \times 10^{5}$ pfu (plaqueforming units)/ml of a derivative (GLV1h73) of previously described GLV1h68 (21). In contrast to GLV1h68, GLV1h73 has no gene insertions in the deleted $F 14.5 \mathrm{~L}$ and $A 56 \mathrm{R}$ loci. After one hour, cells were transfected with transfer vectors using FuGENE 6 transfection reagent (Roche Applied Science, Mannheim, Germany). Recombinant VACV expressing wildtype or mutant Cdc6 were purified by five consecutive rounds of plaque purification in the presence of selective drugs. Virus stocks were prepared on CV-1 cells and further purified by sucrose gradient centrifugation. Viral titers were determined by plaque assays on $\mathrm{CV}-1$ cells.

$R T-P C R$ analyses. At indicated time points total RNA from uninfected and VACV-infected GI-101A cells (MOI 0.5) was prepared using the RNeasy mini kit and the RNase-free DNase set (both from Qiagen, Hilden, Germany) following the manufacturer's instructions. An additional DNase digest was performed with the DNA-free reagent (Ambion, Austin, TX, USA). cDNA synthesis was performed with the Revert Aid First Strand cDNA synthesis kit (Fermentas, St. LeonRot, Germany) in a total volume of $20 \mu \mathrm{l}$ using $1 \mu \mathrm{g}$ of each RNA sample and oligo $(\mathrm{dT})_{18}$ primers according to the protocol provided. Each cDNA sample $(1.5 \mu 1)$ was amplified using Taq DNA polymerase (Fermentas) and primers listed above (Table I). PCR conditions were 2 min at $95^{\circ} \mathrm{C}$, followed by 30 cycles of $30 \mathrm{sec}$ at $95^{\circ} \mathrm{C}, 30 \mathrm{sec}$ at $53^{\circ} \mathrm{C}, 1 \mathrm{~min}$ at $72^{\circ} \mathrm{C}$, and finally $7 \mathrm{~min}$ at $72^{\circ} \mathrm{C}$.

Immunoblot analyses. Protein extracts from GI-101A cells were prepared by the following method: cells were infected at MOI 0.5 or left uninfected. At the time points indicated, cells were washed twice with ice-cold PBS (PAA). Proteins were isolated by scraping cells directly into lysis buffer 
(15 mM NaCl, 1 mM EDTA, 10 mM HEPES, pH 7.4, $1.5 \mathrm{mM}$ DTT, $0.1 \%$ NP-40, protease inhibitors) and passing detached cells through a 20 -gauge $(0.9 \mathrm{~mm})$ needle attached to a syringe for 30 times. After two centrifugation steps (10.000 rpm, $4^{\circ} \mathrm{C}$ ) protein concentrations were determined by Bradford colorimetry. Equal amounts $(20 \mu \mathrm{g})$ of protein were subjected to SDS-PAGE and transferred onto nitrocellulose membranes (Schleicher \& Schuell, Dassel, Germany). Membranes were probed with primary antibodies followed by corresponding secondary antibodies conjugated with horseradish peroxidase and detected by the ECL system (Amersham Biosciences). The following antibodies were used: anti-Cdc6 (CC30, Calbiochem), anti- $\beta$-galactosidase (A-11132, Molecular Probes) and anti- $\beta$-actin (ab6276, Abcam).

Viral replication assay. For the viral replication assay, GI$101 \mathrm{~A}$ cells grown in $24-w e l l$ plates were infected with VACV at MOI 0.1 in infection medium (RMPI-1640 containing $2 \% \mathrm{FBS}$ and supplements). After one hour of incubation at $37^{\circ} \mathrm{C}$ with gentle agitation every $20 \mathrm{~min}$, virus inoculi were removed and replaced by fresh infection medium. After 4, 12, 24, 48 or $72 \mathrm{~h}$, cells and supernatants were harvested. Following three freeze-thaw cycles, serial dilutions of the lysates were titered by standard plaque assays on CV-1 cells. All samples were measured in triplicate.

MTT assay. For quantitation of virus cytotoxity, VACVinfected and uninfected GI-101A cells in 24-well plates $\left(4 \times 10^{4}\right.$ cells/well in triplicate) were analyzed using 3-(4,5dimethylthiazol-2-yl)-2,5-diphenyltetrazolium bromide (MTT) assay. Cells were infected with VACV at MOI 0.5 or mock-infected with infection medium (RMPI-1640 containing $2 \% \mathrm{FBS}$ and supplements). After one hour of incubation, virus inoculi were removed by aspiration and fresh medium was added. After 24, 48 and $72 \mathrm{~h}$, respectively, media were removed and $500 \mu \mathrm{l}$ MTT $(2.5 \mathrm{mg} / \mathrm{ml})$ solution in RMPI without phenol red was added for $4 \mathrm{~h}$ at $37^{\circ} \mathrm{C}$ and $5 \% \mathrm{CO}_{2}$. MTT solution was then removed and $400 \mu \mathrm{l}$ of $1 \mathrm{~N} \mathrm{HCl}$ in isopropanol was added. Each sample $(3 \times 100 \mu 1)$ was transferred to a 96 -well plate and absorbance was measured at $570 \mathrm{~nm}$ with a reference wavelength of $650 \mathrm{~nm}$ in a Sunrise Microplate reader (Tecan, Austria). The percentage of cell survival was calculated using the following formula: \% cell survival = (absorbance value of infected cells/absorbance value of uninfected control cells) $\mathrm{x}$ $100 \%$.

Animal model and treatment. All animal experiments were approved by the government of Unterfranken and conducted according to the German animal protection guidelines. Tumors were generated by implanting $5 \times 10^{6}$ GI-101A cells [in $100 \mu 1$ PBS (PAA)] subcutaneously into the right flank of 6-8-week-old female nude mice (NCI/Hsd/Athymic NudeFoxn $1^{\text {nu }}$, Harlan Winkelmann GmbH, Borchen, Germany). Tumor volume was measured in two dimensions using a digital caliper and calculated with the following formula: [(length $\mathrm{x}$ width $\left.{ }^{2}\right) \mathrm{x}$ 0.52]. When tumors reached $300-500 \mathrm{~mm}^{3}$, mice were infected via tail vein with $5 \times 10^{6}$ pfu of VACV (in $100 \mu 1 \mathrm{PBS}$ ) or PBS as control and tumor growth was monitored weekly.
To assess viral distribution, GI-101A tumor-bearing mice were infected via tail vein with $5 \times 10^{6}$ pfu of VACV (in $100 \mu 1$ PBS). Ten days after infection, mice were sacrificed. Tumors and organs were prepared and homogenized using FastPrep ${ }^{\mathrm{TM}}$ FP120 (Thermo Electron Corporation, Langenselbold, Germany). After three freeze-thaw cycles, viral titers in homogenates were determined by standard plaque assays on $\mathrm{CV}-1$ cells. All samples were measured in duplicate.

\section{Results}

Construction of recombinant VACV expressing wild-type and mutant murine Cdc6 genes. In previous studies it was shown that a recombinant VACV, GLV-1h68, leads to inhibition of tumor growth in different types of human cancers as well as canine mammary cancer (21-28). In this study we wanted to improve the anti-tumor potency of this virus by constructing a derivative which carries a gene for a Walker A motif mutant (K237E) of Cdc6. We expected that delivering a mutated essential cell cycle protein directly into tumors would lead to impaired tumor cell DNA replication and therefore enhanced anti-tumor effects of VACV.

Cdc6 DNA, amplified from plasmids containing wildtype or the K237E mutant of Cdc6, was cloned into VACV $A 56 R$ transfer plasmids under control of a VACV synthetic strong early promoter (PSSE). Transfer vectors were sequenced to ascertain the presence of Cdc6 genes and promoters and to verify the K237E mutation in the mutant Cdc6 transfer plasmid (data not shown). Recombinant VACV were then generated as described in the Materials and methods section. Purified viruses were again sequenced to assure that no additional mutations developed during the procedure (data not shown). A schematic illustration of newly generated recombinant vaccinia viruses, GLV-1h236 and GLV-1h237, is shown in Fig. 1. Wild-type or mutant Cdc6 cDNA under control of the PSSE promoter were inserted into the A56R locus of VACV. These viruses also contain a LacZ expression cassette in the $J 2 R$ locus as well as a functional deletion, i.e. an insertion of a short non-sense sequence, of the $F 14.5 \mathrm{~L}$ locus. Since TFR cDNA is inserted in reverse orientation to the promoter it is not expressed. As control viruses served previously described GLV-1h68 (21) and a derivative thereof, GLV-1h71. The latter one was chosen because GLV-1h71, like the newly generated VACV recombinants, contains two gene expression cassettes in the $J 2 R$ and $A 56 R$ locus and deletion of the $F 14.5 \mathrm{~L}$ locus, respectively.

Recombinant VACV encoding wild-type or mutant Cdc6 overexpress Cdc6 in GI101A cells. In a first set of experiments, we studied the expression of Cdc6 and marker gene lacZ in VACV-infected human breast cancer GI101A cells by RT-PCR and immunoblot analyses. Therefore, GI101A cells were infected with GLV-1h236 or GLV-1h237 at multiplicity of infection (MOI) of 0.5 and total RNA was isolated at indicated time points. Kinetics of Cdc6 mRNA expression was then analyzed by RT-PCR using murine Cdc6-specific primer pairs. Induction of Cdc6 mRNA synthesis was observed as soon as one hour after infection in both GLV-1h236- and GLV-1h237-infected cells (Fig. 2A). This was expected since Cdc6 constructs are under control of 
GLV-1h236

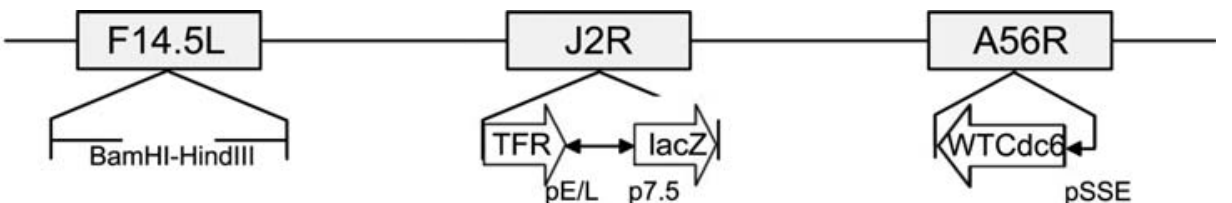

GLV-1h237

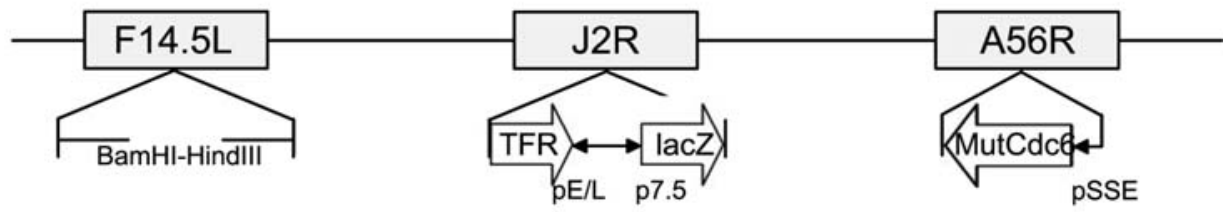

GLV-1h71
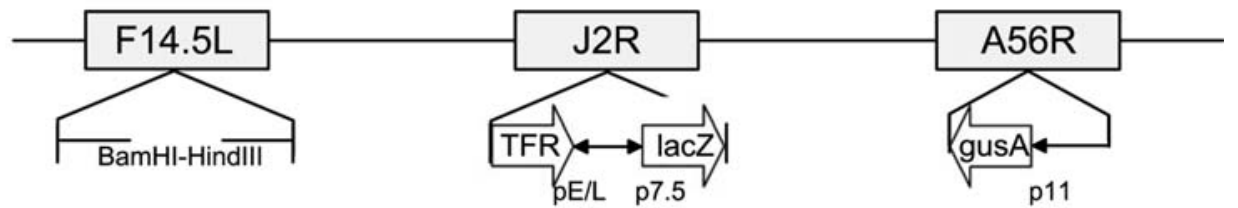

GLV-1h68
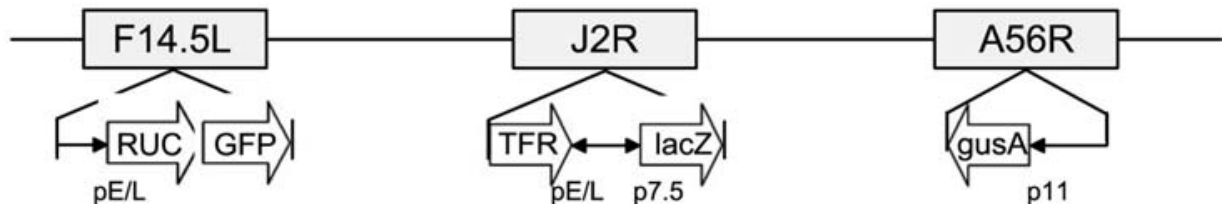

Figure 1. Schematic illustration of VACV used in this study. Genes for murine wild-type and mutant Cdc6 were inserted in the A56R locus of a derivative of previously described VACV GLV-1h68 (21) in which the marker genes RUC-GFP and GusA in the $F 14.5 L$ and A56R loci, respectively, had been removed. GLV1h71 as well as GLV-1h68 served as control viruses. PE/L, VACV synthetic early/late promoter; P7.5, VACV early/late promoter; PSSE, VACV synthetic strong early promoter; $P 11$, VACV late P11 promoter.

A

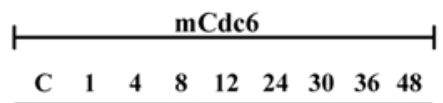

GLV-1h236

$\begin{array}{lllllllll}C & 1 & 4 & 8 & 12 & 24 & 30 & 36 & 48\end{array}$

GLV-1h237

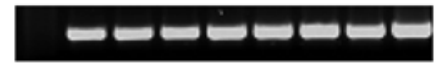

hCde6

GLV-1h71

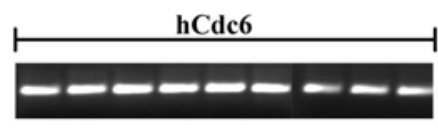

B

GLV-1h236

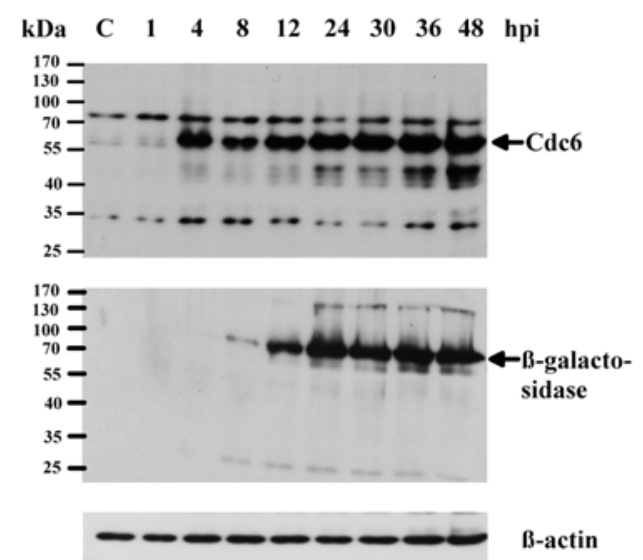

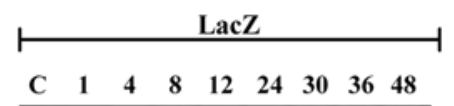
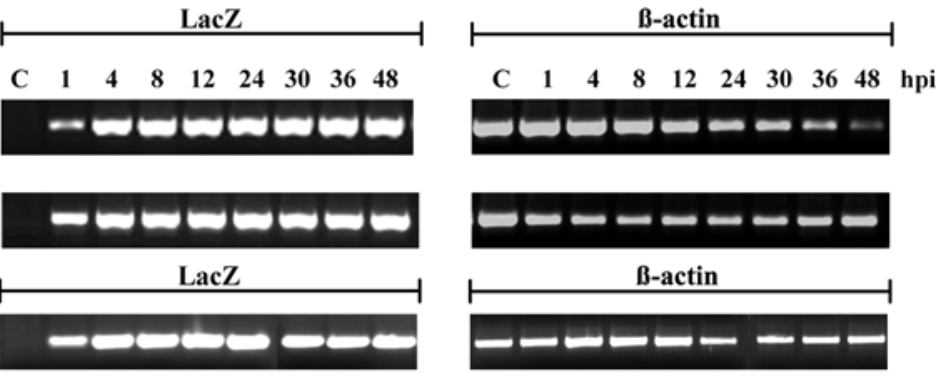

GLV-1h237

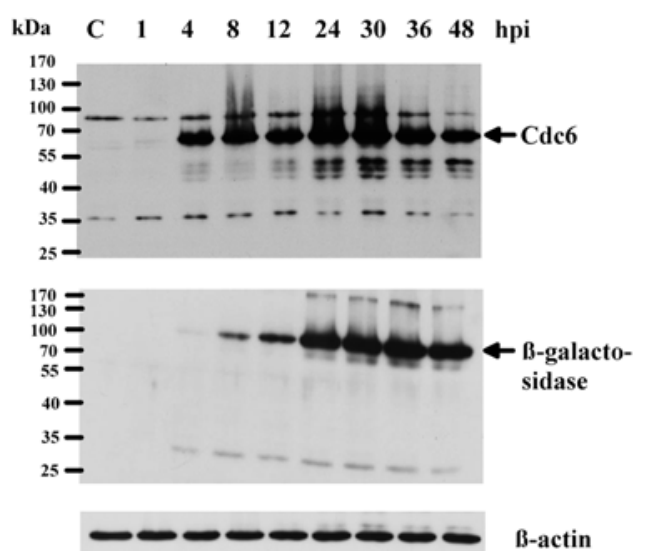

Figure 2. Cdc6 overexpression in GLV-1h236- and GLV-1h237-infected GI-101A cells. (A) Total RNA was isolated at indicated time points from GI-101A cells infected with GLV-1h236, GLV-1h237 and GLV-1h71 (MOI 0.5) and cDNA synthesis was performed. PCRs were carried out with indicated primer pairs. (B) Proteins were extracted at indicated time points from GI-101A cells infected with GLV-1h236 and GLV-1h237 (MOI 0.5). Expression of indicated proteins was determined by immunoblotting. Molecular size markers (kDa) are shown on the left. C, uninfected control; $h p i$, hours post infection; $m C d c 6$, murine Cdc6-specific primer pairs; $h C d c 6$, human $C d c 6$-specific primer pairs. 

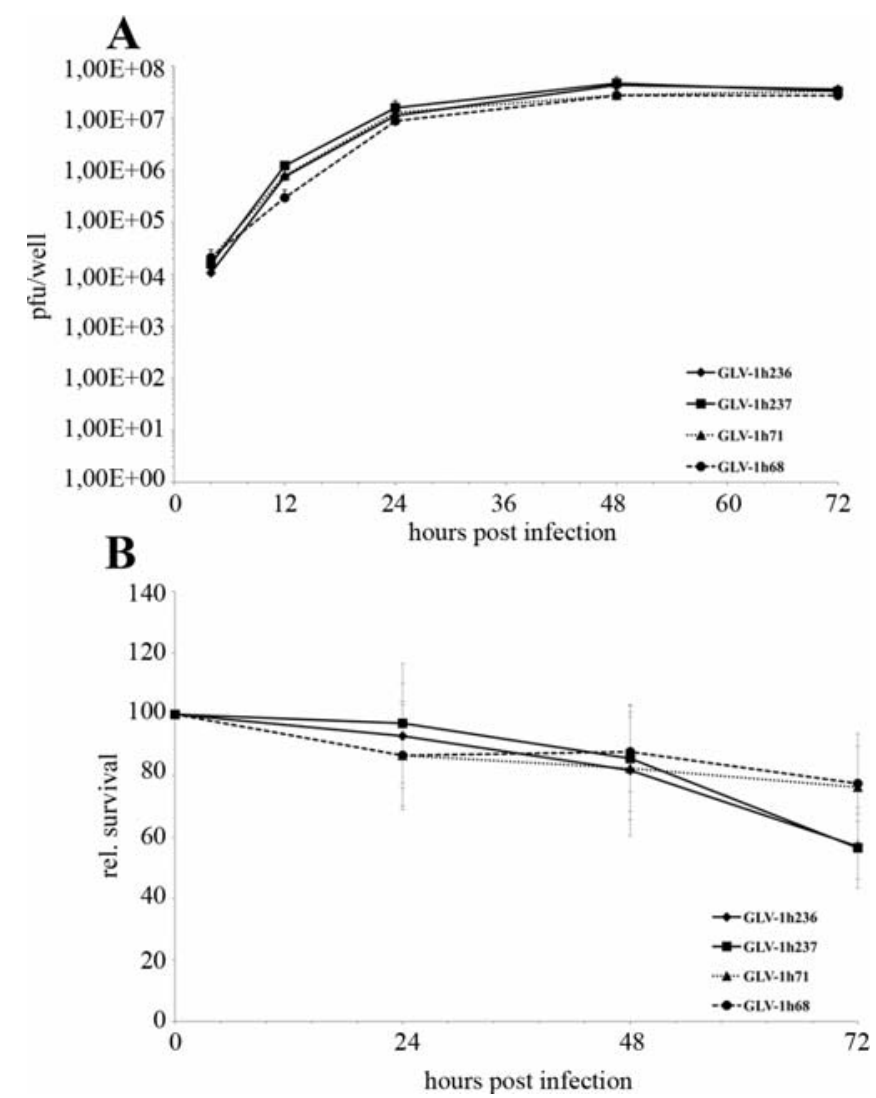

Figure 3. New recombinant VACV efficiently replicate in and lyse human breast cancer GI-101A cells. (A) Replication of different VACV in GI-101A cells infected with MOI 0.1. Cells and supernatants were harvested over time and viral titers were determined as pfu/well in triplicate by standard plaque assays on CV-1 cells. Average plus standard deviation are plotted (B) Viability of GI-101A cells upon VACV infection. Tumor cells were infected with VACV (MOI 0.5) or left uninfected and viable cells were determined using 3-(4,5-dimethylthiazol-2-yl)-2,5-diphenyltetrazolium bromide (MTT) assay. The cell survival compared to untreated cells (in \%) is shown.

a synthetic strong early VACV promoter and early VACV gene expression is known to start within minutes after infection (46). During the infection course, levels of Cdc6 mRNA increased. Expression of marker gene $l a c Z$, under control of an early/late VACV promoter, was also detectable $1 \mathrm{hpi}$. Transcript levels of $\beta$-actin were used as control. To exclude the possibility that the increase of Cdc6 mRNA levels is due to VACV infection per se, synthesis of endogenous Cdc6 mRNA in GLV-1h71-infected cells was analyzed by primer pairs annealing to human Cdc6. As shown in Fig. 2A (lower row), VACV infection has no enhancing effect on endogenous Cdc6 mRNA synthesis. Thus, elevated Cdc6 mRNA levels observed in GLV-1h236- and GLV-1h237-infected cells derive from virus-encoded overexpression.

Cdc6 expression was further confirmed by immunoblot analyses. Proteins were extracted at different time points from GLV-1h236- or GLV-1h237-infected cells (MOI 0.5), separated by SDS-PAGE and immunoblotted for Cdc6 and $\beta$-galactosidase. Membranes were probed with anti- $\beta$-actin as loading control. In GLV-1h236- and GLV-1h237-infected cells, Cdc6 protein overexpression was detectable at 4 hpi. Expression of the marker protein $\beta$-galactosidase could be observed from 8 hpi on. Thus, by RT-PCR and immunoblot analyses it was shown that in GI-101A cells infected with the newly generated VACV recombinants, GLV-1h236 and GLV-1h237, virus-encoded Cdc6 is overexpressed.

Replication and oncolytic effects of GLV-1h236 and GLV1 h237 in cell culture. To determine whether inserted wildtype or mutant Cdc6 genes influence viral proliferation, we quantified production of infectious viral particles over time following infection. For that, GI-101A cells were infected with different VACV at a MOI of 0.1 and total virus yield was determined in lysed cells and supernatants by titering on CV-1 cells. All investigated viruses showed comparable replication kinetics with a 3-log increase in viral titers from 4 to 72 hpi (Fig. 3A). However, if replication multiples are compared (72 hpi versus 4 hpi), GLV-1h236 and GLV-1h237 replicate stronger (3396 resp. 2089) than GLV-1h71 or GLV-1h68 (1680 resp. 1317). Furthermore, after $72 \mathrm{~h}$ higher amounts of viral particles were found in supernatants of GLV-1h236- and GLV-1h237-infected cells (55.78\% resp. $45.81 \%$; i.e. $44.22 \%$ resp. $55.19 \%$ were intracellular or cellassociated) than in supernatants of GLV-1h71- or GLV-1h68infected cells (35.05\% resp. $34.55 \%$; i.e. $64.95 \%$ resp. $65.45 \%$ intracellular or cell-associated) suggesting that GLV-1h236 and GLV-1h237 lyse GI-101A cells more efficiently.

In order to investigate the cytotoxic effects of the viruses more directly, MTT assays were performed. GI-101A cells were infected with VACV (MOI 0.5) or left uninfected and relative cell survival was determined at indicated time points as described in the Materials and methods section. Cell viability was markedly decreased after infection of GI-101A cells with VACV (Fig. 3B). After 72 h, infection with GLV$1 \mathrm{~h} 236$ or GLV-1h237 resulted in $57 \pm 11 \%$ and $57 \pm 13 \%$ cell survival in contrast to $\sim 77 \pm 17 \%$ and $77 \pm 12 \%$ cell survival after infection with GLV-1h71 or GLV-1h68. Thus, GLV$1 \mathrm{~h} 236$ and GLV-1h237 have a stronger oncolytic effect on GI-101A cells compared to control viruses. Together, the higher replication efficiency and the enhanced cytotoxicity of GLV-1h236 and GLV-1h237 on GI-101A cells suggest that these viruses also might have a greater therapeutic potency for treating GI-101A tumors in a mouse model.

GLV-1h237 efficiently colonizes GI-101A tumors in mice. To investigate biodistribution of systemically injected VACV, homogenates of tumors and organs from intravenously injected $\left(5 \times 10^{6} \mathrm{pfu}\right)$ GI-101A tumor-bearing nude mice $(\mathrm{n}=4)$ were titered on CV-1 cells 10 days after injection. All investigated VACV localized primarily to the tumors and were either not detectable or found at notably lower titers in normal tissues (Table II). GLV-1h236-infected tumors displayed the highest viral load, on average $1.5 \times 10^{8} \pm 9.48 \times 10^{7} \mathrm{pfu} / \mathrm{g}$ tumor tissue, followed by GLV-1h71-, GLV-1h237- and GLV-1h68infected tumors, which showed titers of $5.66 \times 10^{7} \pm 1.48 \times 10^{7}$, $3.36 \times 10^{7} \pm 3.49 \times 10^{6}$ and $1.24 \times 10^{7} \pm 9.19 \times 10^{6} \mathrm{pfu} / \mathrm{g}$, respectively. GLV-1h236 and GLV-1h71 were more infective to normal tissues, since these viruses were found at higher titers than GLV-1h237 and GLV1h68 in spleens and lungs. However, the newly generated VACV, GLV-1h237, has comparable safety profiles and tumor selectivity as previously described GLV-1h68. 
Table II. Tumor selectivity of VACV in vivo.

\begin{tabular}{lccr}
\hline & & Mean pfu/g tissue or organ \\
\cline { 2 - 4 } & \multicolumn{1}{c}{ Tumor } & Spleen & Lungs \\
\hline GLV-1h236 & $1.50 \times 10^{8} \pm 9.48 \times 10^{7}$ & $2.00 \times 10^{2} \pm 1.94 \times 10^{2}$ & $2.65 \times 10^{3} \pm 1.92 \times 10^{3}$ \\
GLV-1h237 & $3.36 \times 10^{7} \pm 3.49 \times 10^{6}$ & $62.7 \pm 95.1$ & $8.11 \times 10^{2} \pm 9.81 \times 10^{2}$ \\
GLV-1h71 & $5.66 \times 10^{7} \pm 1.48 \times 10^{7}$ & $71.9 \pm 88.6$ & $1.54 \times 10^{3} \pm 1.09 \times 10^{3}$ \\
GLV-1h68 & $1.24 \times 10^{7} \pm 9.19 \times 10^{6}$ & $65.9 \pm 86.7$ & $1.76 \times 10^{2} \pm 1.84 \times 10^{2}$ \\
\hline
\end{tabular}

GI-101A tumor-bearing nude mice $(\mathrm{n}=4)$ were infected with $5 \times 10^{6}$ pfu of VACV. Ten days after infection, tumors and organs were harvested and viral titers were determined by standard plaque assays on CV-1 cells. Results are shown as mean pfu/g tissue or organ.

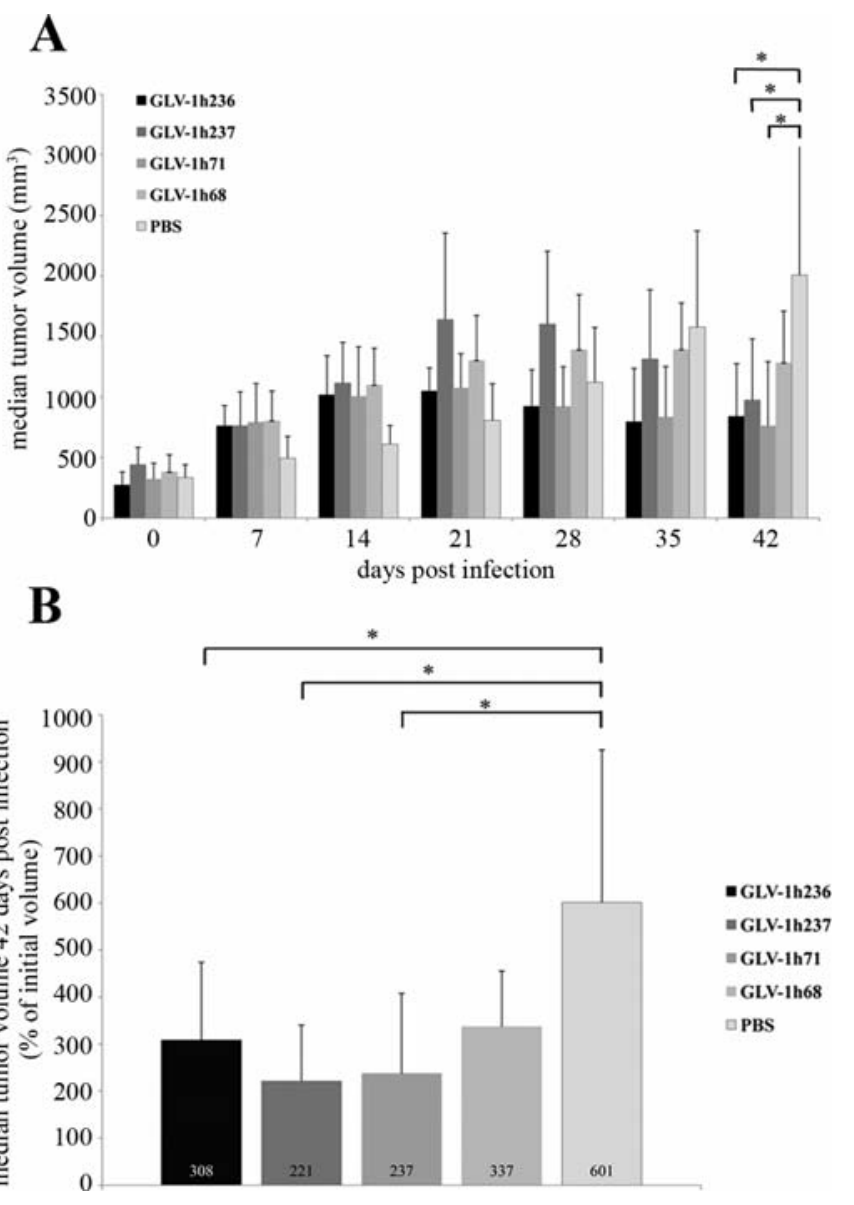

Figure 4. Regression of GI-101A xenografts after VACV infection. (A) Female nude mice bearing GI-101A tumors $\left(300-500 \mathrm{~mm}^{3} ; \mathrm{n}=10\right)$ were infected via tail vein with $5 \times 10^{6}$ pfu of indicated VACV or PBS as control. Average tumor volume per week is shown. A two-tailed Student's t-test was used for statistical analysis. $\mathrm{P}<0.05$ were considered statistically significant. (B) Diagram displaying median tumor volumes 42 days after infection expressed as a percentage of tumor volumes at time of infection (initial volume).

Enhanced therapeutic effect of GLV-1h237 on GI-101A tumors in nude mice. To investigate whether GLV-1h237 has an enhanced anti-tumor activity as formerly applied GLV1h68, nude mice bearing GI-101A xenografts were treated with different VACV. Mice were implanted with $5 \times 10^{6}$ GI-
$101 \mathrm{~A}$ cells into the right flank and tumors were allowed to develop to volumes of $300-500 \mathrm{~mm}^{3}$ before $5 \times 10^{6} \mathrm{pfu}$ of individual VACV or PBS as control were administered via tail vein injection. Tumor growth was then monitored weekly for 42 days. Fig. 4A shows the kinetics of tumor regression after treatment of nude mice with VACV. Control mice injected with PBS showed exponential tumor growth. In marked contrast, infection of mice with VACV led to tumor regression, which was significant in the GLV-1h236-, GLV1h237- and GLV-1h71-treated groups. Volumes of infected tumors peaked at 21 to 28 days followed by tumor regression in all treatment groups. Fig. 4B shows tumor volumes after 42 days normalized to the day of virus injection. Control tumors had a volume of $601 \pm 324 \%$ compared to the beginning of the experiment. Strikingly, treatment of GI-101A tumors with GLV-1h237 led to the most prominent anti-tumor response resulting in $221 \pm 119 \%$ of the initial tumor volume. The anti-tumor effects of GLV-1h236 (308 $\pm 166 \%)$ and GLV-1h71 $(237 \pm 170 \%)$ were slightly better than that of GLV-1h68 (337 $\pm 119 \%)$. These in vivo experiments clearly demonstrated that GLV-1h237 displayed a similar tumor selectivity and safety profile concomitant with an enhanced anti-tumor activity compared to GLV-1h68. Therefore, GLV$1 \mathrm{~h} 237$ might be useful as a novel therapeutic agent for the treatment of cancers.

\section{Discussion}

Oncolytic virotherapy has great potential in the treatment of cancer since the active agent, i.e. the virus, is amplificated preferentially within the tumor but not in normal tissues. By genetically engineering oncolytic viruses, additional diagnostic or therapeutic tools can be delivered directly into tumors. Recently it was reported that a newly constructed recombinant VACV, GLV-1h68, shows an enhanced tumor-targeting specificity and a greatly reduced toxicity compared with its parental strain LIVP (21). A single i.v. injection of GLV$1 \mathrm{~h} 68$ led to tumor regression of subcutaneous GI-101A human breast cancer xenografts. Furthermore, GLV-1h68 was used to successfully treat different types of human cancers as well as canine mammary cancers (22-28). In the present study we intended to further enhance the promising antitumor potential of GLV-1h68 without decreasing its safety. Since replication proteins such as Cdc6, Cdt1 or MCM are 
known to be often overexpressed in cancer cells (40-44), we thought to arm VACV with a mutated replication protein with the aim to interfere with tumor cell DNA replication. It has previously been reported that silencing of replicationinitiating genes, such as $\mathrm{Cdc} 6, \mathrm{Mcm} 2$ or $\mathrm{Cdc} 45$, results in inhibition of DNA replication and cell proliferation as well as p53-independent apoptosis of cancer cells but not of normal cells (47). Especially the induction of p53-independent apoptosis renders the strategy of inhibiting DNA replication proteins for cancer therapy very attractive since approximately half of the cancers are p53-defective.

In the present study, a new recombinant VACV, GLV1h237, starting from a derivative of GLV-1h68, was engineered which contains the murine gene for a Walker A motif mutant of the essential cell cycle protein Cdc6 in order to treat human GI-101A breast tumors. Replication proteins like Cdc6 are highly conserved during eukaryotic evolution (48). We therefore expected that the use of the murine cDNA might not affect the therapeutic efficiency of treating human breast tumors by GLV-1h237. Moreover, in this study we used a mouse model of human breast cancer and we did not observe any enhanced toxicity on mouse organs after delivering of VACV overexpressing murine mutant Cdc6. This implies that GLV-1h237 specifically targets and destroys tumors, even that of human origin, but does not harm normal tissues of the body, an aspect that is very essential for future cancer therapy of patients.

To more thoroughly assess the therapeutic effect of GLV$1 \mathrm{~h} 237$ on human breast cancer, we compared this construct to the first-generation oncolytic VACV GLV-1h68 and in parallel to GLV-1h236, expressing wild-type Ccd6 as well as to GLV-1h71, a VACV that only differs from the newly generated VACVs in that it contains an expression cassette for gusA in the A56R locus instead that for wild-type or mutant Cdc6. RT-PCR and immunoblot analyses revealed that Cdc6 was expressed in human breast cancer GI-101A cells after infection with GLV-1h236 or GLV-1h237. Using anti-Cdc6 antibodies in immunoblot experiments not only the 62-kDA full-length Cdc6 but also smaller fragments were detected. The latter migrate between 40 and $55 \mathrm{kDa}$, especially later in the course of infection (Fig. 2B). It has previously been reported that human Cdc6 is cleaved by caspase 3 during apoptosis $(49,50)$. However, it remains to be investigated if the fragments observed in our immunoblot experiments derive from specific cleavage by caspases during apoptosis or if they result from unspecific cleavage due to massive overexpression in infected cells.

We also observed that GLV-1h236 and GLV-1h237 replicated stronger and had an enhanced oncolytic effect on GI-101A cells compared to GLV-1h68 and GLV-1h71. In vivo, both effects are probably important for efficient spreading of viral particles in tumors and, thus, for the therapeutic potency of the newly generated Cdc6-expressing viruses.

In the in vivo GI-101A tumor xenograft model GLV-1h237 turned out to be the most potent virus investigated. We demonstrated that GLV-1h237 had similarly high tumor selectivity and a comparable safety profile as GLV-1h68 since GLV$1 \mathrm{~h} 237$ localized primarily to the tumors and was either not detectable or found at much lower titers in other organs. A single i.v. injection of $5 \times 10^{6} \mathrm{pfu}$ GLV-1h237 resulted in a tumor volume of $221 \pm 119 \%$ of the initial volume 42 days after infection. In contrast, after infection with GLV-1h68 a tumor volume of $337 \pm 119 \%$ was observed, infection with GLV-1h236 and GLV-1h71 led to a volume of $308 \pm 166 \%$ and $237 \pm 170 \%$, respectively. The molecular events underlying the enhanced tumor regression after infection with GLV-1h237 are currently unknown. Hence one future objective will be to find out how exactly the stronger therapeutic effect of GLV-1h237 is mediated. Wali and Strayer reported that VACV infection leads to an increase in the percentage of cells in $\mathrm{S}$ phase (51). These authors suppose that VACV might benefit from a prolonged $\mathrm{S}$ phase as host cell factors required for or facilitating viral replication may be longer available. Conceivably, blocking the host cell cycle before the onset of $\mathrm{S}$ phase, as it was our aim by using the recombinant GLV-1h237 virus, may contribute to a further more efficient viral proliferation by increasing the time of availability for essential host cell factors.

In conclusion, in the present study it was shown that GLV-1h237 has a stronger anti-tumor activity compared to previously used GLV-1h68 concomitant with high tumor selectivity and comparable safety profile. However, this work did not elucidate the effects of overexpressed mutant Cdc6 on tumor cell cycle machinery directly. Therefore, future studies have to elucidate the molecular mechanisms of how GLV-1h237 mediates the enhanced therapeutic effect on GI$101 \mathrm{~A}$ breast cancer xenografts. However, the data reported here suggest the application of GLV-1h237 as a novel improved agent for oncolytic cancer therapy.

\section{Acknowledgements}

We thank Qian Zhang and Nanhai Chen for their generous help in virus construction, Johanna Langbein and Christina Tietze for excellent technical support and Ivaylo Gentschev and Jochen Stritzker for critically reading the manuscript. This work was supported by grants from Genelux Corporation (R\&D facility in San Diego, CA, USA). Elisabeth Hofmann is a recipient of a postdoctoral fellowship by Genelux Corporation awarded to the University of Würzburg.

\section{References}

1. Heise CC, Williams AM, Xue S, Propst M and Kirn DH: Intravenous administration of ONYX-015, a selectively replicating adenovirus, induces antitumoral efficacy. Cancer Res 59: 2623-2628, 1999.

2. Galanis E, Okuno SH, Nascimento AG, et al: Phase I-II trial of ONYX-015 in combination with MAP chemotherapy in patients with advanced sarcomas. Gene Ther 12: 437-445, 2005.

3. Peng KW, TenEyck CJ, Galanis E, Kalli KR, Hartmann LC and Russell SJ: Intraperitoneal therapy of ovarian cancer using an engineered measles virus. Cancer Res 62: 4656-4662, 2002.

4. Heinzerling L, Kunzi V, Oberholzer PA, Kundig T, Naim H and Dummer R: Oncolytic measles virus in cutaneous T-cell lymphomas mounts antitumor immune responses in vivo and targets interferon-resistant tumor cells. Blood 106: 2287-2294, 2005.

5. Balachandran S, Porosnicu M and Barber GN: Oncolytic activity of vesicular stomatitis virus is effective against tumors exhibiting aberrant $\mathrm{p} 53$, Ras, or myc function and involves the induction of apoptosis. J Virol 75: 3474-3479, 2001.

6. Stojdl DF, Lichty BD, ten Oever BR, et al: VSV strains with defects in their ability to shutdown innate immunity are potent systemic anti-cancer agents. Cancer Cell 4: 263-275, 2003. 
7. Pecora AL, Rizvi N, Cohen GI, et al: Phase I trial of intravenous administration of PV701, an oncolytic virus, in patients with advanced solid cancers. J Clin Oncol 20: 2251-2266, 2002.

8. Phuangsab A, Lorence RM, Reichard KW, Peeples ME and Walter RJ: Newcastle disease virus therapy of human tumor xenografts: antitumor effects of local or systemic administration. Cancer Lett 172: 27-36, 2001

9. Coffey MC, Strong JE, Forsyth PA and Lee PW: Reovirus therapy of tumors with activated Ras pathway. Science 282: 1332-1334, 1998.

10. Hirasawa K, Nishikawa SG, Norman KL, et al: Systemic reovirus therapy of metastatic cancer in immune-competent mice. Cancer Res 63: 348-353, 2003.

11. Fu X and Zhang X: Potent systemic antitumor activity from an oncolytic herpes simplex virus of syncytial phenotype. Cancer Res 62: 2306-2312, 2002.

12. Nakamori M, Fu X, Pettaway CA and Zhang X: Potent antitumor activity after systemic delivery of a doubly fusogenic oncolytic herpes simplex virus against metastatic prostate cancer. Prostate 60: 53-60, 2004.

13. Shen $Y$ and Nemunaitis J: Fighting cancer with vaccinia virus: teaching new tricks to an old dog. Mol Ther 11: 180-195, 2005.

14. Park BH, Hwang T, Liu TC, et al: Use of a targeted oncolytic poxvirus, JX-594, in patients with refractory primary or metastatic liver cancer: a phase I trial. Lancet Oncol 9: 533-542, 2008 .

15. Thorne SH, Bartlett DL and Kirn DH: The use of oncolytic vaccinia viruses in the treatment of cancer: a new role for an old ally? Curr Gene Ther 5: 429-443, 2005.

16. McCart JA, Ward JM, Lee J, et al: Systemic cancer therapy with a tumor-selective vaccinia virus mutant lacking thymidine kinase and vaccinia growth factor genes. Cancer Res 61 : 8751-8757, 2001.

17. Guo ZS, Naik A, O'Malley ME, et al: The enhanced tumor selectivity of an oncolytic vaccinia lacking the host range and anti-apoptosis genes SPI-1 and SPI-2. Cancer Res 65: 99919998, 2005

18. Kirn DH, Wang Y, Le Boeuf F, Bell J and Thorne SH: Targeting of interferon-beta to produce a specific, multi-mechanistic oncolytic vaccinia virus. PLoS Med 4: e353, 2007.

19. Thorne SH, Hwang TH, O'Gorman WE, et al: Rational strain selection and engineering creates a broad-spectrum, systemically effective oncolytic poxvirus, JX-963. J Clin Invest 117 3350-3358, 2007.

20. Hung CF, Tsai YC, He L, et al: Vaccinia virus preferentially infects and controls human and murine ovarian tumors in mice. Gene Ther 14: 20-29, 2007.

21. Zhang Q, Yu YA, Wang E, et al: Eradication of solid human breast tumors in nude mice with an intravenously injected lightemitting oncolytic vaccinia virus. Cancer Res 67: 10038-10046, 2007.

22. Lin SF, Price DL, Chen CH, et al: Oncolytic vaccinia virotherapy of anaplastic thyroid cancer in vivo. J Clin Endocrinol Metab 93: 4403-4407, 2008.

23. Kelly KJ, Woo Y, Brader P, et al: Novel oncolytic agent GLV1 h68 is effective against malignant pleural mesothelioma. Hum Gene Ther 19: 774-782, 2008

24. Yu Z, Li S, Brader P, et al: Oncolytic vaccinia therapy of squamous cell carcinoma. Mol Cancer 8: 45, 2009.

25. Yu YA, Galanis C, Woo Y, et al: Regression of human pancreatic tumor xenografts in mice after a single systemic injection of recombinant vaccinia virus GLV-1h68. Mol Cancer Ther 8: 141-151, 2009.

26. Gentschev I, Donat U, Hofmann E, et al: Regression of human prostate tumors and metastases in nude mice following treatment with the recombinant oncolytic vaccinia virus GLV-1h68. J Biomed Biotechnol: 489759, 2010.

27. Gentschev I, Stritzker J, Hofmann E, et al: Use of an oncolytic vaccinia virus for the treatment of canine breast cancer in nude mice: preclinical development of a therapeutic agent. Cancer Gene Ther 16: 320-328, 2008.

28. Gentschev I, Ehrig K, Donat U, et al: Significant growth inhibition of canine mammary carcinoma xenografts following treatment with oncolytic vaccinia virus GLV-1h68. J Oncol: 736907,2010 .
29. Takisawa H, Mimura S and Kubota Y: Eukaryotic DNA replication: from pre-replication complex to initiation complex. Curr Opin Cell Biol 12: 690-696, 2000.

30. Bell SP and Dutta A: DNA replication in eukaryotic cells. Annu Rev Biochem 71: 333-374, 2002.

31. Neuwald AF, Aravind L, Spouge JL and Koonin EV: AAA ${ }^{+}$: A class of chaperone-like ATPases associated with the assembly, operation, and disassembly of protein complexes. Genome Res 9: 27-43, 1999.

32. Story RM and Steitz TA: Structure of the recA protein-ADP complex. Nature 355: 374-376, 1992.

33. Saraste M, Sibbald PR and Wittinghofer A: The P-loop - a common motif in ATP- and GTP-binding proteins. Trends Biochem Sci 15: 430-434, 1990.

34. Walker JE, Saraste M, Runswick MJ and Gay NJ: Distantly related sequences in the alpha- and beta-subunits of ATP synthase, myosin, kinases and other ATP-requiring enzymes and a common nucleotide binding fold. EMBO J 1: 945-951, 1982.

35. Wang B, Feng L, Hu Y, et al: The essential role of Saccharomyces cerevisiae CDC6 nucleotide-binding site in cell growth, DNA synthesis, and Orc1 association. J Biol Chem 274: 8291-8298, 1999.

36. Weinreich M, Liang C and Stillman B: The Cdc6p nucleotidebinding motif is required for loading $\mathrm{mcm}$ proteins onto chromatin. Proc Natl Acad Sci USA 96: 441-446, 1999.

37. Herbig U, Marlar CA and Fanning E: The Cdc6 nucleotidebinding site regulates its activity in DNA replication in human cells. Mol Biol Cell 10: 2631-2645, 1999.

38. Cook JG, Park CH, Burke TW, et al: Analysis of Cdc6 function in the assembly of mammalian prereplication complexes. Proc Natl Acad Sci USA 99: 1347-1352, 2002.

39. Frolova NS, Schek N, Tikhmyanova N and Coleman TR: Xenopus Cdc6 performs separate functions in initiating DNA replication. Mol Biol Cell 13: 1298-1312, 2002.

40. Gonzalez MA, Pinder SE, Callagy G, et al: Minichromosome maintenance protein 2 is a strong independent prognostic marker in breast cancer. J Clin Oncol 21: 4306-4313, 2003.

41. Semple JW and Duncker BP: ORC-associated replication factors as biomarkers for cancer. Biotechnol Adv 22: 621-631, 2004.

42. Karakaidos P, Taraviras S, Vassiliou LV, et al: Overexpression of the replication licensing regulators hCdt1 and hCdc6 characterizes a subset of non-small-cell lung carcinomas: synergistic effect with mutant p53 on tumor growth and chromosomal instability-evidence of E2F-1 transcriptional control over hCdt1. Am J Pathol 165: 1351-1365, 2004.

43. Williams GH, Romanowski P, Morris L, et al: Improved cervical smear assessment using antibodies against proteins that regulate DNA replication. Proc Natl Acad Sci USA 95: 14932-14937, 1998.

44. Xouri G, Lygerou Z, Nishitani H, Pachnis V, Nurse P and Taraviras S: Cdt1 and geminin are down-regulated upon cell cycle exit and are over-expressed in cancer-derived cell lines. Eur J Biochem 271: 3368-3378, 2004.

45. Faul T: Localization and dynamics of replication proteins of the murine pre-replicative complex. $\mathrm{PhD}$ thesis, University of Wuerzburg, 2004

46. Broyles SS: Vaccinia virus transcription. J Gen Virol 84: 2293-2303, 2003.

47. Feng D, Tu Z, Wu W and Liang C: Inhibiting the expression of DNA replication-initiation proteins induces apoptosis in human cancer cells. Cancer Res 63: 7356-7364, 2003.

48. Kelly TJ and Brown GW: Regulation of chromosome replication. Annu Rev Biochem 69: 829-880, 2000.

49. Yim H, Hwang IS, Choi JS, et al: Cleavage of Cdc6 by caspase-3 promotes ATM/ATR kinase-mediated apoptosis of HeLa cells. J Cell Biol 174: 77-88, 2006.

50. Pelizon C, d'Adda di Fagagna F, Farrace L and Laskey RA: Human replication protein Cdc6 is selectively cleaved by caspase 3 during apoptosis. EMBO Rep 3: 780-784, 2002.

51. Wali A and Strayer DS: Infection with vaccinia virus alters regulation of cell cycle progression. DNA Cell Biol 18: 837-843, 1999. 